\title{
Development of vesicovaginal fistula caused by vaginal adenosis: A case report
}

\author{
Tasuku Mariya $^{1}$, Takahiro Suzuki ${ }^{1,2}$, Shutaro Habata ${ }^{1}$, Motoki Matsuura ${ }^{1}$, Miwa Suzuki ${ }^{1}$, \\ Ryoichi Tanaka', Tsuyoshi Saito ${ }^{1}$ \\ ${ }^{1}$ Department of Obstetrics and Gynecology, Sapporo Medical University, Sapporo, Japan \\ ${ }^{2}$ Department of Obstetrics and Gynecology, Tokeidai Hospital, Sapporo, Japan \\ Email: t.mariya0725@gmail.com
}

Received 16 May 2013; revised 14 June 2013; accepted 21 June 2013

Copyright (c) 2013 Tasuku Mariya et al. This is an open access article distributed under the Creative Commons Attribution License, which permits unrestricted use, distribution, and reproduction in any medium, provided the original work is properly cited.

\begin{abstract}
Introduction: Vaginal adenosis is one of the rare diseases of the vagina, and almost all patients are asymptomatic. We report a case of spontaneous vaginal adenosis, which caused vesicovaginal fistula. Case Presentation: Our patient was a 25-year-old Japanese woman. She was admitted to our hospital, and her chief complaint was continuous urine flow from the vagina. We found a tumor with a vesicovaginal fistula in her vagina. Subsequent cytological analysis of vaginal smears showed a normal vaginal mucosa. The patient underwent tumor resection and a fistula patch. Pathological diagnosis was adenosis of the vagina, even though the patient had no known history of intrauterine diethylstilbestrol exposure or Müllerian developmental abnormalities. Conclusion: The clinical course of our case was a malignant tumor, which invasively bored a hole in the vaginal wall forming a vesicovaginal fistula, even though it was a benign lesion. Therefore, overzealous treatment should be avoided in this case.
\end{abstract}

Keywords: Vaginal Adenosis; Vesicovaginal Fistula; Surgical Repair; Urine Continence

\section{INTRODUCTION}

We describe a rare case involving a benign vaginal tumor with a vesicovaginal fistula. Although the tumor had been growing with enlargement of the vesicovaginal fistula, the pathological diagnosis was adenosis with no malignancy. This is the first case report of vaginal adenosis showing such an invasive development and bored vesicovaginal fistula. We describe how we treated this case successfully with surgery.

\section{CASE PRESENTATION}

The patient was a 25-year-old nulligravida Japanese woman. She had a regular menstrual cycle and there was nothing of particular note in her past history. Although the patient had never previously experienced incontinence, persistent urine leakage from her vagina started in December 2009. Because of a gradual increase in this incontinence, she consulted our hospital in April 2010. Magnetic resonance imaging suggested that there was a tumor approximately $2 \mathrm{~cm}$ in diameter that had developed from the vaginal wall, and a vaginal fistula right beside the tumor (Figure 1). Cystoscopic examination showed a fistula in the middle of the trigone, and incontinent urine flow from the fistula into vagina. In the vaginal speculum, the fistula was located in the deeper side of a polyp and urine leakage continued from the fistula. Intravaginal biopsy showed that the histology of the tumor was normal vaginal tissue. We suspected that the tumor was malignant because of the gradually increasing volume of the incontinent urine flow and enlargement of the fistula. One month after the patient first visited our hospital, we carried out an operation. We approached the bladder in an abdominal operation through the space of Retzius. First, we incised the bladder longitudinally and checked the status of the vesicovaginal fistula. Despite the fact that the tumor was located close to the internal urine meatus, fortunately, we were able to keep a margin of at least $5 \mathrm{~mm}$. We then cut the bladder to the vaginal wall surrounding the tumor as a whole, extending out to the vaginal cavity. We closed the hole in the bladder and anterior vaginal wall caused by resection of the tumor by suturing it layer-by-layer. The final pathological diagnosis of the lesion was vaginal adenosis. There was a difference between the first partial biopsy and the final pathology. The reason for this conflicting result could have been because of the patient's 


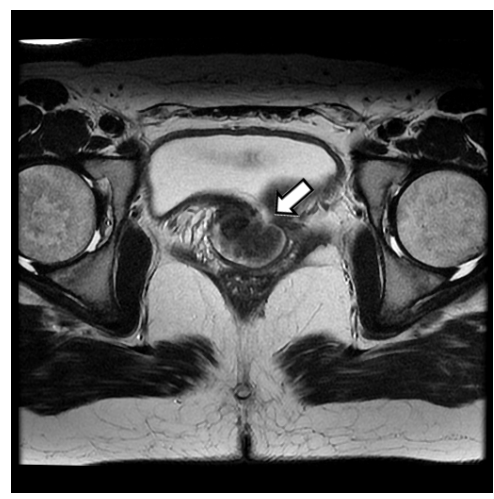

Figure 1. Magnetic resonance imaging of the vesicovaginal fistula. MRI shows that the fistula had bored a hole through the vaginal wall beside the polyp (arrow). The tensioned wall of the bladder extending into the polyp is clearly visible.

vagina was narrow and the vaginal wall was tensioned by adenosis. Therefore, the major involvement around the fistula, which was screened by the polyp, was too deep to permit harvesting of a sufficient amount of tissue.

\section{DISCUSSION}

Generally, a vesicovaginal fistula can be caused by injury to the urinary tract, which can occur accidentally during surgery to the pelvic area, such as during a hysterectomy. A vesicovaginal fistula can also be caused by a malignant tumor in the vesicovaginal area, or by reduced blood supply due to necrosis caused by radiation therapy or prolonged labor during childbirth [1]. In our case, because there was a tumor with a vesicovaginal fistula, we suspected that the fistula was caused by the malignant tumor. Histological examination of the complete specimen resected in the operation showed the presence of columnar epithelium on the surface and stroma of the tumor. The vaginal tumor formed small islands of columnar epithelium that were similar to the columnar epithelium of the cervix (Figure 2). Spontaneous vaginal adenosis is thought to be derived from a persistent Müllerian epithelium islet during postembryonic life [2]. This disease is also known to be induced by drugs, such as diethylstilbestrol (DES). There are many reports that women are at high risk of adenosis and vaginal clear cell carcinoma after exposure to intrauterine DES or 5fluorouracil. In women prenatally exposed to DES, vaginal adenosis may appear in up to $90 \%$ of cases [2]. However, our patient could not be given intrauterine DES because the use of DES was prohibited in Japan before her mother became gravid. Almost all recent reports regarding vaginal adenosis were about DES exposed cases. Therefore, epidemiological knowledge of this disease, which occurs spontaneously, such as in our

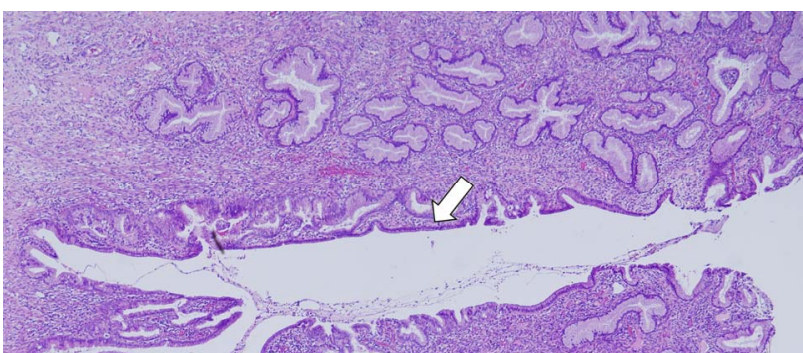

Figure 2. Histology of part of the fistula. A part of the branched gland is enlarged and an especially large cavity of the gland can be seen, which constitutes part of the vesicovaginal fistula (arrow). The site of transition, from squamous to glandular epithelium, is sudden and sharply defined. Hematoxylin/eosin stain at $40 \times$ magnification.

case, is poor. There are some reports on the occurrence of vaginal adenocarcinoma from vaginal adenosis, which has no association with prenatal DES exposure. In one case report, a patient with vaginal clear cell adenocarcinoma had congenital malformation of the genital tract, including a bicornuate uterus, vaginal septum, and left ureteral agenesis [3]. In another report, the author showed two cases in which gastric type mucinous adenocarcinoma occurred from vaginal adenosis. In addition, one of these cases had uterus didelphys [4]. These two case reports suggest a probable causal association between congenital malformation, vaginal adenosis, and vaginal adenocarcinoma. Commonly reported complaints for adenosis are abnormal vaginal bleeding, dyspareunia, a feeling of heat in the vagina, and mucous discharge. A definitive diagnosis of adenosis is difficult to make from symptoms and a gross examination. Previous studies have been performed regarding gross examination of adenosis, which was associated with DES exposure. Stafl et al. [5] reported 63 young women whose mothers were given DES during the first trimester of pregnancy. Vaginal adenosis was found in $91 \%$ of these women, and $71 \%$ had no visible changes. The remaining $29 \%$ presented with a vaginal hood, cervix with a cock's comb appearance, and erythroplakia in a gross examination.

\section{CONCLUSION}

Although our patient showed a developing vesicovaginal fistula and the pore gradually enlarged, the lesion was not malignant. Therefore, we were able to treat the adenosis completely with resection of the polyp and closure of the fistula. In this disease, we can conduct a definitive diagnosis only by histological analysis. Therefore, overzealous treatment should not be performed, especially when surgical treatment is chosen.

\section{REFERENCES}

[1] Narayanan, P., Nobbenhuis, M., Reynolds, K.M., Sahdev, 
A., Reznek, R.H. and Rockall, A.G. (2009) Fistulas in malignant gynecologic disease: Etiology, imaging, and management. RadioGraphics, 29, 1073-1083. doi:10.1148/rg.294085223

[2] Kranl, C., Zelger, B., Kofler, H., Heim, K., Sepp, N. and Fritsch, P. (1998) Vulvar and vaginal adenosis. British Journal of Dermatology, 139, 128-131. doi:10.1046/j.1365-2133.1998.02329.x

[3] Uehara, T., Onda, T., Sasajima, Y., Sawada, M. and Kasamatsu, T. (2010) A case of vaginal clear cell adenocarcinoma complicated with congenital anomalies of the genitourinary tract and metanephric remnant without prenatal diethylstilbestrol exposure. Journal of Obstetrics and Gynaecology Research, 36, 681-685.

[4] Talia, K.L., Scurry, J., Manolitsas, T. and McCluggage, W.G. (2012) Primary vaginal mucinous adenocarcinoma of gastric type arising in adenosis: A report of 2 cases, 1 associated with uterus didelphys. International Journal of Gynecological Pathology, 31, 184-191. doi:10.1097/PGP.0b013e31822c8036

[5] Stafl, A., Mattingly, R.F., Foley, D.V. and Fetherston, W.C. (1974) Clinical diagnosis of vaginal adenosis. $\mathrm{Ob}$ stetrics and Gynecology, 43, 118-128. 